\title{
Free energies for rare events: Temperature accelerated MD and MC
}

\author{
S. Meloni ${ }^{1, a}$ and G. Ciccotti ${ }^{2, b}$
}

1 Laboratory of Computational Chemistry and Biochemistry, Institut des Sciences et Ingénierie Chimiques, École Polytechnique Fédérale de Lausanne, 1015 Lausanne, Switzerland

2 Dipartimento di Fisica and CNISM, Università La Sapienza, P. le A. Moro 5, 00185 Rome, Italy; School of Physics, University College Dublin, Belfield, Dublin, Ireland

Received 20 March 2015 / Received in final form 5 May 2015

Published online 22 June 2015

\begin{abstract}
In this article we review a set of methods for exploring the space of a set of collective variables, and to reconstruct the associated Landau free energy in presence of metastabilities: Temperature Accelerated Molecular Dynamics (TAMD), its extension, Temperature Accelerate Monte Carlo (TAMC), and the Single Sweep Method (SSM). TAMD and TAMC can be used for both exploring and reconstructing the Landau free energy landscape. However, SSM is more efficient at accomplishing this last task. We illustrate the use of these methods by presenting their application to the nucleation of a Lennard-Jones crystal from its melt, and the $\mathrm{H}$-vacancy migration in an $\mathrm{NaAlH}_{6}$ crystal.
\end{abstract}

\section{Introduction}

In this article we review a set of methods for studying rare events by atomistic simulations. Rare events, which we will define more precisely below, are infrequent transitions between high probable states of a system. From the point of view of statistical mechanics rare events are like any other (more frequent) event. The difference stands in the simulation techniques that must be used to investigate them: because of their low frequency we cannot use standard (brute force) molecular dynamics (MD) or Monte Carlo (MC). To put rare events in their more general theoretical context, and to introduce quantities that are relevant for their description, in this introduction we start revising the basic ideas behind statistical mechanics.

Let us consider a classical system made of $N$ point particles. A microscopic state of this system is described by a point in the $6 N$-dimensional phase space $\boldsymbol{\Gamma}=(\boldsymbol{r}, \boldsymbol{p})$. $\boldsymbol{r}$ and $\boldsymbol{p}$ are the $3 N$-dimensional vectors of the positions and momenta of the particles. Consider an observable $\mathcal{O}(\boldsymbol{\Gamma})$. The corresponding macroscopic value is the time average of the observable:

$$
\overline{\mathcal{O}}=\lim _{t \rightarrow \infty} \frac{1}{t} \int_{0}^{t} d \tau \mathcal{O}(\boldsymbol{\Gamma}(\tau)),
$$

\footnotetext{
a e-mail: simone.meloni@epfl.ch

b e-mail: giovanni.ciccotti@roma1.infn.it
} 
i.e. the average of $\mathcal{O}(\boldsymbol{\Gamma})$ along the trajectory $\boldsymbol{\Gamma}(\tau)$ of the system. Assuming that the system is ergodic, the time average is equivalent to the ensemble average over the probability density function (PDF) $m(\boldsymbol{\Gamma})$ of the system. This is easily seen by discretizing the time trajectory, expressing the time integral as a sum over these discrete times, rearranging the terms of the sum according to their assignment to cells of a discretization of the phase space, and transforming back this discrete sum into an integral

$$
\begin{aligned}
\overline{\mathcal{O}}=\lim _{\mathcal{M} \rightarrow \infty} \frac{1}{\mathcal{M} h} \sum_{i=1}^{\mathcal{M}} \mathcal{O}\left(\boldsymbol{\Gamma}_{i}\right) h & =\lim _{\mathcal{M} \rightarrow \infty} \lim _{\mathcal{N} \rightarrow \infty} \sum_{s=1}^{\mathcal{N}} \frac{n_{s}}{\mathcal{M}} \mathcal{O}\left(\boldsymbol{\Gamma}_{s}\right) \\
& =\int d \Gamma \mathcal{O}(\boldsymbol{\Gamma}) m(\boldsymbol{\Gamma})=\langle\mathcal{O}\rangle
\end{aligned}
$$

The sum in the third term of the equality runs over the states associated to the discretized phase space.

Any mechanical property of the system can be computed as an expectation value of a suitable microscopic observable over the PDF of the system. $m(\boldsymbol{\Gamma})$, then, is the central non mechanical quantity of statistical mechanics. In thermodynamics the same role is played by the thermodynamics potentials, $A(\boldsymbol{X})$ (with $\boldsymbol{X}$ the set of thermodynamic variables): any property of the system can be obtained by suitable analytical operations (e.g. derivatives with respect to $\mathbf{X}$ ) on the relevant thermodynamic potential: entropy, free energies, etc, depending on the independent thermodynamics variables considered. Thus, it is natural to draw an equivalence between $m(\boldsymbol{\Gamma})$ and $A(\boldsymbol{X})$. In the microcanonical Boltzmann formula of the entropy (variables $\mathrm{N}, \mathrm{V}$, E), $S=k_{B} \log Q$ with $Q$ partition function, this equivalence does not emerge clearly. However, remembering the hypothesis of equiprobability of the accessible microscopic states, this formula can be rewritten so as to make it evident:

$$
S=k_{B} \log Q=-k_{B} \log (1 / Q) \equiv-\log \left(m_{N V E}(\boldsymbol{\Gamma})\right)
$$

where the subscript $N V E$ stresses that $m_{N V E}(\boldsymbol{\Gamma})=1 / Q$, with $Q$ geometrical measure of the set of accessible states (constant energy surface), is the PDF of the microscopic states of the microcanonical system (within accessible phase space). We call the averages of observables mechanical properties and quantities derived from $m(\boldsymbol{\Gamma})$, including marginal and conditional PDFs, thermal properties. Thus, $m(\boldsymbol{\Gamma})$ itself determines the entire family of the thermal properties of the system.

Let us now come back to the calculation of $\langle\mathcal{O}\rangle$. Equation (2) can be rewritten as:

$$
\begin{aligned}
\langle\mathcal{O}\rangle & =\int d \boldsymbol{\Gamma} \mathcal{O}(\boldsymbol{\Gamma}) m(\boldsymbol{\Gamma})=\int d \mathcal{O}^{*} \mathcal{O}^{*} \int d \boldsymbol{\Gamma} \delta\left(\mathcal{O}(\boldsymbol{\Gamma})-\mathcal{O}^{*}\right) m(\boldsymbol{\Gamma}) \\
& =\int d \mathcal{O}^{*} \mathcal{O}^{*} p_{\mathcal{O}}\left(\mathcal{O}^{*}\right)
\end{aligned}
$$

where $p_{\mathcal{O}}\left(\mathcal{O}^{*}\right)=\int d \boldsymbol{\Gamma} \delta\left(\mathcal{O}(\boldsymbol{\Gamma})-\mathcal{O}^{*}\right) m(\boldsymbol{\Gamma})$ is the reduced, or marginal, PDF of the observable $\mathcal{O}(\boldsymbol{\Gamma})$. The information on $\mathcal{O}(\boldsymbol{\Gamma})$ contained in $p_{\mathcal{O}}\left(\mathcal{O}^{*}\right)$ is obviously contained also in $m(\boldsymbol{\Gamma})$. However, due to the high dimensionality of the phase space, with $m(\boldsymbol{\Gamma})$ this does not emerge clearly. So, when one is interested in the values of a specific observable, $\mathcal{O}(\boldsymbol{\Gamma})$, or any function of it, $p_{\mathcal{O}}\left(\mathcal{O}^{*}\right)$ is the central quantity. In analogy with what done with the total PDF, we introduce the quantity

$$
W_{\mathcal{O}}\left(\mathcal{O}^{*}\right)=-k_{B} \log p_{\mathcal{O}}\left(\mathcal{O}^{*}\right)
$$


The analogy between Eqs. (5) and (3) suggests that $W_{\mathcal{O}}\left(\mathcal{O}^{*}\right)$ is a restriction to $\mathcal{O}^{*}$ of the thermodynamic potential. In App. A we prove that $\Delta W_{\mathcal{O}}=W_{\mathcal{O}}\left(\mathcal{O}_{2}\right)-W_{\mathcal{O}}\left(\mathcal{O}_{2}\right)$ is the reversible work made by a generalized force to move the system from the state $\mathcal{O}(\boldsymbol{\Gamma})=\mathcal{O}_{1}$ to $\mathcal{O}(\boldsymbol{\Gamma})=\mathcal{O}_{2}$, confirming that $W_{\mathcal{O}}\left(\mathcal{O}^{*}\right)$ is, indeed, a free energy. A final $\mathrm{PDF}$ that is important to introduce is the conditional PDF

$$
m\left(\boldsymbol{\Gamma} \mid \mathcal{O}^{*}\right)=\frac{\delta\left(\mathcal{O}(\boldsymbol{\Gamma})-\mathcal{O}^{*}\right) m(\boldsymbol{\Gamma})}{p_{\mathcal{O}}\left(\mathcal{O}^{*}\right)}
$$

$m\left(\boldsymbol{\Gamma} \mid \mathcal{O}^{*}\right)$ allows to compute the expectation of an observable at a given value of $\mathcal{O}(\boldsymbol{\Gamma}):\left\langle\mathcal{B} \mid \mathcal{O}^{*}\right\rangle=\int d \boldsymbol{\Gamma} \mathcal{B}(\boldsymbol{\Gamma}) m\left(\boldsymbol{\Gamma} \mid \mathcal{O}^{*}\right)$.

Summarizing, the central quantities in statistical mechanics are the total or reduced PDF, depending on whether one is interested on the entire phase space or only on an (or few, see Sect. 2) observable. To each of these PDFs is associated a suitable thermodynamic potential, from which one can derive all the properties of the corresponding thermodynamic system. In addition, the conditional PDF $m\left(\boldsymbol{\Gamma} \mid \mathcal{O}^{*}\right)$ allows to compute conditional expectation values.

To introduce rare events, we start by classifying the total PDF in two classes. In the first class we put monomodal PDFs and multimodal PDFs in which the regions between the modes have a relatively high probability density. The second class contains multimodal PDFs in which the modes are separated by domains of very low probability, with a difference of several orders of magnitudes between the values of the PDF of the modes and of the regions separating them. A trajectory takes relatively short time to visit the various high probable regions of PDFs belonging to the first class. So, brute force MD, of the typical duration of few picoseconds, up to microseconds, is suitable to sample PDFs of this kind, and compute unbiased expectation values of observables through Eq. (2). In the case of PDFs of the second kind, the passage of the system from the region of one mode to another is infrequent, because it has to pass through the low probable region in between them. These infrequent events are the rare events we focus on in this article. In presence of rare events the residence time in the region of one mode is so long with respect to the duration of MD simulations, that the brute force approach is unsuited to properly sample the PDFs.

In many cases, the metastable states of a system, and the passage from one to another, can be described in terms of selected observables and the associated reduced PDF. We can apply to this PDF the above analysis on the structure of the the total PDF: when two modes of the reduced PDF of the relevant observables are separated by a minimum of very low probability the trajectory takes long time to pass from one to the other, and Brute force MD is inadequate to sample it.

The problem of rare events has been known for very long time, and some analytical and numerical techniques have been introduced long time ago to deal with it. For example, the termodynamics integration of Kirkwood dates back to the mid-thirties [21], even before modern computers were introduced. Another example is the umbrella sampling of Torrie and Valleau, introduced in the late seventies [48]. Nevertheless, many problems remain unaddressed. For example how to deal with the case of reduced PDFs of several/many observables, or how to identify their modes when little is known in advance (identification of stable and metastable states). The methods we discuss in this article, the Temperature Accelerated Molecular Dynamics (TAMD [28]) and Monte Carlo (TAMC [4] - see also Ref. [52] and Refs. $[36,39])$ and the Single Sweep method (SSM $[29,32]$ ) are attempts to solve these problems. They share some ingredients with other methods emerged in the same period, such as the Local Elevation Method [17], and the closely related Conformational Flooding [13] and Metadynamics [23], and the Canonical Adiabatic Free Energy 
Sampling (CAFES [51]). The reader might want to refer to these articles to have a more complete knowledge of the field.

The article is organized as follows. In Sect. 2 we present the methods listed above (TAMD, TAMC and SSM). In Sect. 3 we illustrate the use of the above methods through some application. Finally, in Sect. 4 we draw conclusions. As already mentioned above, the article contains also an appendix, App. A, in which we prove that $W_{\mathcal{O}}\left(\mathcal{O}^{*}\right)$ is a free energy.

\section{Methods}

In this section we describe three related methods, Temperature Accelerated Molecular Dynamics (TAMD [28]), the associated Temperature Accelerated Monte Carlo (TAMC [4]), and the Single Sweep method (SSM) [29,32], aimed at exploring and computing the free energy of a set of observables, also called collective variables (CVs). We consider the case of CVs that are function of the particles position only, $\boldsymbol{\xi}(\boldsymbol{r})=\left\{\xi_{i}(\boldsymbol{r})\right\}_{i=1, M}$. The reduced PDF of $\boldsymbol{\xi}(\boldsymbol{r})$ is a straightforward extension of the one of a single observable introduced in Sect. 1.

$$
p_{\boldsymbol{\xi}}(\boldsymbol{z})=\int d \boldsymbol{r} m(\boldsymbol{r}) \prod_{i=1, M} \delta\left(\xi_{i}(\boldsymbol{r})-z_{i}\right)
$$

where $\boldsymbol{z}=\left\{z_{i}\right\}_{i=1, M}$ is a set of realizations of the CVs, and $m(\boldsymbol{r})=\int d \boldsymbol{p} m(\boldsymbol{r}, \boldsymbol{p})$ is the PDF of a point $\boldsymbol{r}$ of the configuration subspace. To $p_{\boldsymbol{\xi}}(\boldsymbol{z})$ is associated a Landau free energy, $W_{\boldsymbol{\xi}}(\boldsymbol{z})=-k_{B} T \log p_{\boldsymbol{\xi}}(\boldsymbol{z})$.

\subsection{Temperature accelerated molecular dynamics and temperature accelerated Monte Carlo}

TAMD and TAMC are two methods aimed at exploring the CV-space, and sampling the associated marginal PDF $p_{\boldsymbol{\xi}}(\boldsymbol{z})$.

With the aim of making more clear the foundations of these methods, we describe them following an unconventional presentation. First (Sect. 2.1.1) we show that the gradient of the Landau free energy, $\boldsymbol{F}_{\boldsymbol{\xi}}(\boldsymbol{z})=\boldsymbol{\nabla} W_{\boldsymbol{\xi}}(\boldsymbol{z})$, can be expressed as the expectation value of a suitable set of observables over the PDF associated to a biased potential depending parametrically on $\boldsymbol{z}, \tilde{V}(\boldsymbol{r} ; \boldsymbol{z})$. This expectation value can be computed by a MD (MC) driven by $\tilde{V}(\boldsymbol{r} ; \boldsymbol{z})$. Even though this method for calculating $\boldsymbol{F}_{\boldsymbol{\xi}}(\boldsymbol{z})$ is just a technical detail of TAMC and TAMD, it is important for the development of the algorithms. Second (Sect. 2.1.2), we introduce a two-steps algorithm for an efficient sampling of $p_{\boldsymbol{\xi}}(\boldsymbol{z})$. In this algorithm, the CVs evolve according to an enhanced dynamics driven by $\boldsymbol{F}_{\boldsymbol{\xi}}(\boldsymbol{z})$, and a biased MD/MC is used to compute $\boldsymbol{F}_{\boldsymbol{\xi}}(\boldsymbol{z})$ at the current (fixed) values of $\boldsymbol{z}$. Third (Sect. 2.1.3), we introduce a one-step algorithm, improving over the previous two-steps one, in which the CVs and the particles are evolved together.

\subsubsection{Calculation of $F_{\boldsymbol{\xi}}(\boldsymbol{z})$ via biased $\mathrm{MD} / \mathrm{MC}$}

From the the Landau free energy of a set of CVs, $W_{\boldsymbol{\xi}}(\boldsymbol{z})$, we can compute

$$
\boldsymbol{F}_{\boldsymbol{\xi}}(\boldsymbol{z})=\nabla_{\boldsymbol{z}} W_{\boldsymbol{\xi}}(\boldsymbol{z})=-k_{B} T \frac{\boldsymbol{\nabla}_{\boldsymbol{z}} p_{\boldsymbol{\xi}}(\boldsymbol{z})}{p_{\boldsymbol{\xi}}(\boldsymbol{z})} .
$$


We show that $\boldsymbol{F}_{\boldsymbol{\xi}}(\boldsymbol{z})$ is the expectation value, conditional to given values of the $\mathrm{CVs}$, of the (partial) derivative of the Hamiltonian, written in terms of the CVs (see App. A). Thus, $-\boldsymbol{F}_{\boldsymbol{\xi}}(\boldsymbol{z})$ can be interpreted as a "mean force". Here we show how $\boldsymbol{F}_{\boldsymbol{\xi}}(\boldsymbol{z})$ can be computed by a more direct approach, replacing the Dirac $\delta$ functions contained in the definition of the marginal probability $p_{\boldsymbol{\xi}}(\boldsymbol{z})$ with a smooth Gaussian approximation:

$$
\delta\left(\xi_{i}(\boldsymbol{r})-z_{i}\right) \sim \sqrt{\frac{\beta \kappa}{2 \pi}} \exp \left[-\beta \frac{\kappa}{2}\left(\xi_{i}(\boldsymbol{r})-z_{i}\right)^{2}\right] .
$$

In Eq. (9) $\beta=1 / k_{B} T$ and $\kappa$ is a parameter controlling the smoothness of the approximation. The Gaussian approximation of the Dirac $\delta$, Eq. (9), can be plugged into Eq. (7) obtaining:

$$
\tilde{p}_{\boldsymbol{\xi}}^{\boldsymbol{\kappa}}(\boldsymbol{z})=\int d \boldsymbol{r} m(\boldsymbol{r}) \prod_{i=1, M} \sqrt{\frac{\beta \kappa_{i}}{2 \pi}} \exp \left[-\beta \frac{\kappa_{i}}{2}\left(\xi_{i}(\boldsymbol{r})-z_{i}\right)^{2}\right] .
$$

In Eq. (10), and in the following, the bold symbol $\kappa$ denotes the vector of elements $\kappa_{i}$. We introduce $\tilde{W}_{\boldsymbol{\xi}}^{\boldsymbol{\kappa}}(\boldsymbol{z})=-k_{B} T \log \tilde{p}_{\boldsymbol{\xi}}^{\kappa}(\boldsymbol{z})$. In view of the fact that in the limit $\left\{\kappa_{i} \rightarrow \infty\right\}_{i=1, M} \tilde{p}_{\boldsymbol{\xi}}^{\boldsymbol{\kappa}}(\boldsymbol{z}) \rightarrow p_{\boldsymbol{\xi}}(\boldsymbol{z})$ and $\tilde{W}_{\boldsymbol{\xi}}^{\boldsymbol{\kappa}}(\boldsymbol{z}) \rightarrow W_{\boldsymbol{\xi}}(\boldsymbol{z})$, we can compute the gradient of the Landau free energy as $\tilde{F}_{\boldsymbol{\xi}}^{\kappa}(\boldsymbol{z})$ in the limit $\boldsymbol{\kappa} \rightarrow \infty$ :

$$
\begin{aligned}
F_{\boldsymbol{\xi}, i}^{\boldsymbol{\kappa}}(\boldsymbol{z}) & =\lim _{\boldsymbol{\kappa} \rightarrow \infty} \frac{\partial W_{\boldsymbol{\xi}}^{\boldsymbol{\kappa}}(\boldsymbol{z})}{\partial z_{i}} \\
& =\lim _{\boldsymbol{\kappa} \rightarrow \infty} \frac{\int d \boldsymbol{r} \kappa_{i}\left(\xi_{i}(\boldsymbol{r})-z_{i}\right) m(\boldsymbol{r}) \prod_{i=1, M} \exp \left[-\beta \frac{\kappa_{i}}{2}\left(\xi_{i}(\boldsymbol{r})-z_{i}\right)^{2}\right]}{\int d \boldsymbol{r} m(\boldsymbol{r}) \prod_{i=1, M} \exp \left[-\beta \frac{\kappa_{i}}{2}\left(\xi_{i}(\boldsymbol{r})-z_{i}\right)^{2}\right]} .
\end{aligned}
$$

In other words, the elements of the gradient of the free energy can be expressed as the limit $\boldsymbol{\kappa} \rightarrow \infty$ of the conditional expectation value of the observable $\kappa_{i}\left(\xi_{i}(\boldsymbol{r}, \boldsymbol{p})-z_{i}\right)$.

We proceed our analysis by considering a specific ensemble, the canonical ensemble: $m(\boldsymbol{r})=\exp [-\beta V(\boldsymbol{r})] / \int d \boldsymbol{r} \exp [-\beta V(\boldsymbol{r})]$, with $V(\boldsymbol{r})$ potential energy. In this ensemble Eq. (11) reads:

$$
F_{\boldsymbol{\xi}, i}(\boldsymbol{z})=\lim _{\boldsymbol{\kappa} \rightarrow \infty} \frac{\int d \boldsymbol{r} \kappa_{i}\left(\xi_{i}(\boldsymbol{r})-z_{i}\right) \exp \left\{-\beta\left[V(\boldsymbol{r})+\sum_{i=1, M} \frac{\kappa_{i}}{2}\left(\xi_{i}(\boldsymbol{r})-z_{i}\right)^{2}\right]\right\}}{\int d \boldsymbol{r} \exp \left\{-\beta\left[V(\boldsymbol{r})+\sum_{i=1, M} \frac{\kappa_{i}}{2}\left(\xi_{i}(\boldsymbol{r})-z_{i}\right)^{2}\right]\right\}} .
$$

The ensemble average at the r.h.s. of Eq. (12) can be estimated by MD or MC driven by the potential $\tilde{V}(\boldsymbol{r} ; \boldsymbol{z})=V(\boldsymbol{r})+\sum_{i=1, M} \frac{\kappa_{i}}{2}\left(\xi_{i}(\boldsymbol{r})-z_{i}\right)^{2}$, as a "time" average of the observable $\kappa_{i}\left(\xi_{i}(\boldsymbol{r})-z_{i}\right)$ along the MD/MC simulation. Thus, by performing an MD/MC simulation with the biased potential $\tilde{V}(\boldsymbol{r} ; \boldsymbol{z})$ and the $\boldsymbol{k}$ large enough we can obtain an estimate for the gradient of the Landau free energy.

\subsubsection{Two-steps algorithm for sampling $p_{\boldsymbol{\xi}}(\boldsymbol{z})$}

Consider the following equation of motion (EoM) for the CVs:

$$
\gamma_{\boldsymbol{z}} \dot{\boldsymbol{z}}=-\boldsymbol{F}_{\boldsymbol{\xi}}(\boldsymbol{z})+\sqrt{2 \bar{\beta}^{-1} \gamma} \boldsymbol{\eta}_{\boldsymbol{z}}(t)
$$




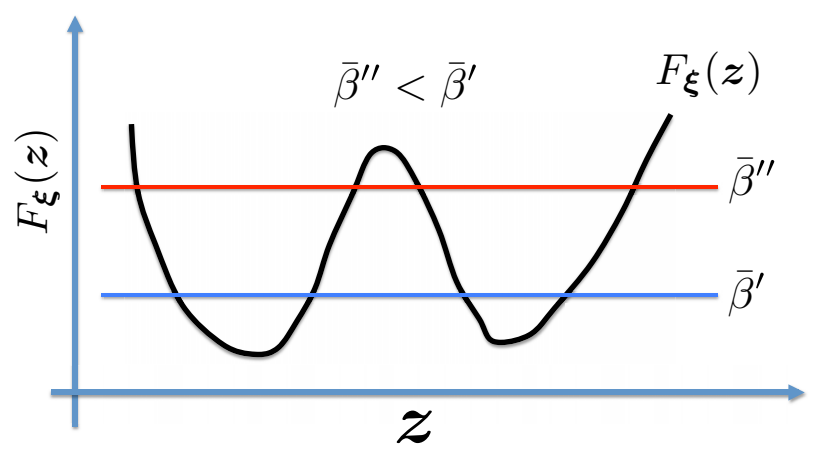

Fig. 1. Sketch of the Landau free energy vs its collective variable $\xi$. The blue and red lines denote the value of the thermal energy at $\bar{\beta}^{\prime}$ and $\bar{\beta}^{\prime \prime}$, respectively. In the case of $\bar{\beta}^{\prime \prime}$ it is easier for the system to overcome the Landau free energy barrier separating the two metastable states and, thus, sampling $\bar{p}_{\boldsymbol{\xi}}(\boldsymbol{z})$.

where $\gamma_{\boldsymbol{z}}$ is a friction coefficient, $\boldsymbol{\eta}_{\boldsymbol{z}}(t)$ is a vector whose elements, $\eta_{i}(t)$, are standard white noise processes, and $\bar{\beta}$ is an inverse temperature that might differ from the physical inverse temperature, $\beta$. The distribution sampled by the dynamics of Eq. (13) is $\bar{p}_{\boldsymbol{\xi}}(\boldsymbol{z})=\exp \left[-\bar{\beta} W_{\boldsymbol{\xi}}(\boldsymbol{z})\right]$, which can be computed by building the histogram of $\boldsymbol{z}$ along the trajectory. $\bar{p}_{\boldsymbol{\xi}}(\boldsymbol{z})$ and $p_{\boldsymbol{\xi}}(\boldsymbol{z})$ are in a very simple relation with each other: $p_{\boldsymbol{\xi}}(\boldsymbol{z})=\bar{p}_{\boldsymbol{\xi}}(\boldsymbol{z})^{\beta / \bar{\beta}}$. Thus, once $\bar{p}_{\boldsymbol{\xi}}(\boldsymbol{z})$ is known it is, in principle, an easy matter to obtain $p_{\boldsymbol{\xi}}(\boldsymbol{z})$.

If $\bar{\beta} \ll \beta$ the value of $\bar{p}_{\boldsymbol{\xi}}(\boldsymbol{z})$ in regions of low probability is exponentially enhanced. Thus, in an MD run the time the system takes to move from one metastable state to another is reduced, and the sampling of the multimodal marginal PDF is faster. This effect can be understood pictorially considering that $\bar{\beta}^{-1}$ is the thermal energy available to the system. When $\bar{\beta}^{-1}$ is much lower than the Landau free energy barrier separating two metastable states the system cannot easily pass from one to the other (see Fig. 1). On the contrary, when the thermal energy is larger, i.e. $\bar{\beta}$ is smaller, the system can easily cross this barrier.

The dynamics of Eq. (13) can be implemented by a two-step algorithm. In the first step the gradient of the Landau Free energy, $\boldsymbol{F}_{\boldsymbol{\xi}}(\boldsymbol{z})$, is computed by RMD or RMC driven by the potential $\tilde{V}(\boldsymbol{r} ; \boldsymbol{z})$ of Sect. 2.1.1 at the current CVs position. In the second step $\boldsymbol{z}$ is evolved according to a time discretized version of Eq. (13) using $\boldsymbol{F}_{\boldsymbol{\xi}}(\boldsymbol{z})$ computed in the first step.

It is worth remarking that any dynamics sampling a PDF of the generalized Boltzmann weight type, $p_{\boldsymbol{\xi}}(\boldsymbol{z})=\exp \left[-\beta W_{\boldsymbol{\xi}}(\boldsymbol{z})\right]$, such as Langevin Dynamics, Nosé-Hoover chanins [30], etc., can be used in place of Eq. (13).

In the following we explain how the calculation of $\boldsymbol{F}_{\boldsymbol{\xi}}(\boldsymbol{z})$ and evolution of $\boldsymbol{z}$ can be merged in a single-step algorithm.

\subsubsection{One-step TAMD/TAMC algorithm}

We can take advantage of the properties of systems composed of sub-systems whose evolution is adiabatically separated (see Ref. [50] and references cited therein) to derive a single-step algorithm to sample $\bar{p}_{\boldsymbol{\xi}}(\boldsymbol{z})$, i.e. an algorithm in which we evolve together $\boldsymbol{z}$ and $\boldsymbol{\Gamma}$. 
Let us start recalling a theorem on the dynamics of adiabatically separated subsystems. Consider a system driven by the following EoM:

$$
\left\{\begin{array}{l}
\dot{y}=\frac{1}{\epsilon} h(x, y) \\
\dot{x}=g(x, y)
\end{array}\right.
$$

where $g(x, y)$ and $h(x, y)$ are the forces governing the dynamics of $x$ and $y$, respectively, and are assumed to be of the same order of magnitude. $\epsilon$ is a parameter measuring the separation of the time-scales in the system. If the dynamics of $y$ at $x$ fixed has an invariant measure, $m_{\epsilon}(y \mid x)$, then in the limit $\epsilon \rightarrow 0 x$ evolves according to the dynamics:

$$
\dot{x}=G(x)
$$

where $G(x)=\lim _{\epsilon \rightarrow 0} \int d y g(x, y) m_{\epsilon}(y \mid x)$. In other words, in the limit $\epsilon \rightarrow 0 x$ evolves according to the effective force $G(x)$.

In TAMD we take advantage of this result to introduce a combined $\boldsymbol{z}-\boldsymbol{r}$ adiabatically separated dynamics in which $\boldsymbol{z}$ moves according to the force $-\boldsymbol{F}_{\boldsymbol{\xi}}(\boldsymbol{z})$. Consider the following EoM:

$$
\left\{\begin{array}{l}
\dot{\boldsymbol{r}}=-\gamma_{\boldsymbol{r}}^{-1} \nabla_{\boldsymbol{r}} \tilde{V}(\boldsymbol{r} ; \boldsymbol{z})+\sqrt{2 \beta^{-1} \gamma_{\boldsymbol{r}}^{-1}} \boldsymbol{\eta}_{\boldsymbol{r}}(t) \\
\dot{\boldsymbol{z}}=-\gamma_{\boldsymbol{z}}^{-1} \boldsymbol{\nabla}_{\boldsymbol{z}} \tilde{V}(\boldsymbol{r} ; \boldsymbol{z})+\sqrt{2 \bar{\beta}^{-1} \gamma_{\boldsymbol{z}}^{-1}} \boldsymbol{\eta}_{\boldsymbol{z}}(t) .
\end{array}\right.
$$

The values of $\gamma_{\boldsymbol{r}}$ and $\gamma_{\boldsymbol{z}}$ can be tuned such that the dynamics of $\boldsymbol{z}$ is much slower than the dynamics of $\boldsymbol{r}$. In practice, the values of $\gamma_{\boldsymbol{r}}$ and $\gamma_{\boldsymbol{z}}$ must be such that $\boldsymbol{z}$ does not move (significantly) over the timescale necessary for the particles' dynamics to sample the conditional PDF $m(\boldsymbol{r} \mid \boldsymbol{z})$. If this condition is met, according to Eqs. (14) and (15) $\boldsymbol{z}$ moves following the effective EoM:

$$
\dot{\boldsymbol{z}}=-\gamma_{\boldsymbol{z}}^{-1} \boldsymbol{G}(\boldsymbol{z})+\sqrt{2 \bar{\beta}^{-1} \gamma_{\boldsymbol{z}}^{-1}} \boldsymbol{\eta}_{\boldsymbol{z}}(t)
$$

with

$$
\begin{aligned}
G_{i}(\boldsymbol{z}) & =\frac{\int d \boldsymbol{r} \partial \tilde{V}(\boldsymbol{r} ; \boldsymbol{z}) / \partial z_{i} \exp [-\beta \tilde{V}(\boldsymbol{r} ; \boldsymbol{z})]}{\int d \boldsymbol{r} \exp [-\beta \tilde{V}(\boldsymbol{r} ; \boldsymbol{z})]} \\
& \equiv \frac{\int d \boldsymbol{r} \kappa_{i}\left(\xi_{i}(\boldsymbol{r})-z_{i}\right) \exp [-\beta \tilde{V}(\boldsymbol{r} ; \boldsymbol{z})]}{\int d \boldsymbol{r} \exp [-\beta \tilde{V}(\boldsymbol{r} ; \boldsymbol{z})]}=F_{\boldsymbol{\xi}, i}^{\boldsymbol{\kappa}}(\boldsymbol{z}) .
\end{aligned}
$$

If the values of $\boldsymbol{\kappa}$ are large enough $\boldsymbol{z}$ moves according to an overdamped dynamics driven by the force $\boldsymbol{F}_{\boldsymbol{\xi}}(\boldsymbol{z})$, thus sampling, without any need to compute explicitly $\boldsymbol{F}_{\boldsymbol{\xi}}(\boldsymbol{z})$, the same PDF, $\bar{p}_{\boldsymbol{\xi}}(\boldsymbol{z})=\exp \left[-\bar{\beta} W_{\boldsymbol{\xi}}(\boldsymbol{z})\right]$, of the two-step algorithm presented in Sect. 2.1.2.

In the original article, Ref. [28], TAMD has been introduced using a set of coupled overdamped EoM (analogous to Eq. (16)). However, this method is not limited to this type of dynamics, and the overdamped dynamics can be straightforwardly replaced by other types of dynamics sampling the same ensemble, for example Langevin dynamics or Nosé-Hoover chains EoM [30] for the canonical ensemble. TAMC [4] takes advantage of this freedom to deal with the case of CVs whose dependence on the 
configuration is not given by an analytical expression. TAMD, in fact, requires that the CVs are differentiable, so that one can compute the forces acting on particles, $-\boldsymbol{\nabla}_{\boldsymbol{r}} \tilde{V}(\boldsymbol{r} ; \boldsymbol{z})=-\left(\boldsymbol{\nabla}_{\boldsymbol{r}} V(\boldsymbol{r})+\sum_{i=1}^{M} \kappa_{i}\left(\xi_{i}(\boldsymbol{r})-z_{i}\right) \boldsymbol{\nabla}_{\boldsymbol{r}} \xi_{i}(\boldsymbol{r})\right)$. On the contrary, MC, which does not require the calculation of forces, can deal with non-differentiable CVs. Thus, we can replace the stochastic dynamics (first row in Eq. (16)) with a MC evolution for the particles. In TAMC, like in TAMD, the evolution of particles is driven by the biased potential $\tilde{V}(\boldsymbol{r} ; \boldsymbol{z})$. The condition for the adiabatical separation of the evolution of $\boldsymbol{z}$ with respect to $\boldsymbol{r}$ can be easily redefined in TAMC: the condition is that $\boldsymbol{z}$ does not move (significantly) over the number of steps necessary for the MC "evolution" of the particles to sample the conditional PDF $m(\boldsymbol{r} \mid \boldsymbol{z})$.

\subsection{The single sweep method}

TAMD and TAMC sample the marginal PDF $\bar{p}_{\boldsymbol{\xi}}(\boldsymbol{z})=\exp \left[-\bar{\beta} W_{\boldsymbol{\xi}}(\boldsymbol{z})\right]$. Thus we could bin the $\boldsymbol{z}$-space and build the corresponding histogram along a TAMD/TAMC trajectory to obtain $\bar{p}_{\boldsymbol{\xi}}(\boldsymbol{z})$. From this we could get $p_{\boldsymbol{\xi}}(\boldsymbol{z})$ by the exponential rescaling:

$$
p_{\boldsymbol{\xi}}(\boldsymbol{z})=\bar{p}_{\boldsymbol{\xi}}(\boldsymbol{z})^{\beta / \bar{\beta}} \text {. }
$$

This approach presents a problem. Given the exponential scaling between the two PDFs, the error on $p_{\boldsymbol{\xi}}(\boldsymbol{z})$ is proportional to the error on $\bar{p}_{\boldsymbol{\xi}}(\boldsymbol{z})$ according to the relation $\sigma_{p_{\boldsymbol{\xi}}(z)} \propto \bar{p}_{\boldsymbol{\xi}}(z)^{\frac{\beta}{\beta}-1} \sigma_{\bar{p}_{\boldsymbol{\xi}}(z)}$. In presence of a rare event $\beta \gg \bar{\beta}$, thus $\beta / \bar{\beta}-1 \sim \beta / \bar{\beta}$, and the previous relation reads $\sigma_{p_{\boldsymbol{\xi}}(z)} \sim \bar{p}_{\boldsymbol{\xi}}(z)^{\beta / \bar{\beta}} \sigma_{\bar{p}_{\boldsymbol{\xi}}(z)}=p_{\boldsymbol{\xi}}(z) \sigma_{\bar{p}_{\boldsymbol{\xi}}(z)}$. This suggests that TAMD and TAMC magnify the error of the estimated reduced PDF with respect to the biased PDF they sample in the regions of high probability, i.e. near the modes of the reduced PDF. Thus, long trajectories are necessary to estimate accurately the reduced PDF. More in general, methods based on binning-and-histogramming require long trajectories to have good statistics on the PDF of the relevant region of the $\mathrm{CV}$ space, and their computational cost scales exponentially with the dimensionality of the space. The objective of the Single Sweep Method (SSM) is to avoid this shortcomings.

Before discussing in detail the method, let us highlight the basic idea. In the SSM the Landau free energy is expanded on a suitable basis set, and the values of the coefficients of the expansion are determined by a least square fitting over the gradient of the Landau free energy obtained from simulations. Two technical problems need to be solved to implement this idea: i) how to select suitable points in the CV-space where to compute the gradient of the Landau free energy for the fitting, and ii) how to chose the basis set for the expansion. In the following, we present the general idea thoroughly, and discuss the technical points in detail.

The SSM algorithm consists of two phases. In the first phase the CV space is explored to identify metastable states and the regions among them. This is the part of the CV space where reactive trajectories tend to pass through, and over which one typically want to reconstruct the Landau free energy landscape. This exploration of the CV space can be performed by TAMD, TAMC or any other accelerating technique. In the second phase we reconstruct the Landau free energy on the reactive region identified in the first phase. We assume that the Landau free energy can be efficiently expanded on a suitable basis set:

$$
\tilde{W}_{\boldsymbol{\xi}}(\boldsymbol{z} ; \boldsymbol{c}, \boldsymbol{\pi})=\sum_{\alpha} c_{\alpha} \chi_{\alpha}(z ; \boldsymbol{\pi})
$$

The basis set, in turn, can depend on few parameters, $\pi=\left\{\pi_{\beta}\right\}_{\beta}$. The values of expansion coefficients, $\boldsymbol{c}=\left\{c_{\alpha}\right\}_{\alpha}$, and the basis set parameters, $\boldsymbol{\pi}$, are obtained by 
fitting $\nabla_{\boldsymbol{z}} \tilde{F}_{\boldsymbol{\xi}}(\boldsymbol{z} ; \boldsymbol{c}, \boldsymbol{\pi})$ on the the estimates gradient of the Landau free energy obtained by RMD/RMC (Sect. 2.1.1) at a suitable set of $\boldsymbol{z}$-points.

The name Single Sweep refers to the fact that, at variance with other methods, here the accelerated trajectory needs to sweep through the CV space only once.

As basis set for the expansion one cannot use "diffuse" functions, like planewaves, as these typically require a regular domain of definition. Thus, their use would require to reconstruct the free energy over a regular subspace of the entire CV-space, an approach that would not scale conveniently with the dimensionality of the CV-space. Localized basis sets suits better the SSM. One typical choice is to use a set of Gaussian functions centered at $\boldsymbol{z}_{\alpha}$ points of the the CV-space: $\chi_{\alpha}(\boldsymbol{z} ; \boldsymbol{\sigma})=\exp \left[-\frac{\left(\boldsymbol{z}-\boldsymbol{z}_{\alpha}\right)^{2}}{2 \sigma_{\alpha}^{2}}\right]$. To define the basis set we must set the value of $\boldsymbol{\sigma}=\left\{\sigma_{\alpha}\right\}_{\alpha}$, a set of tunable parameters that we will discuss shortly, and the centers of the gaussian functions, $\boldsymbol{z}_{\alpha}$. The $\boldsymbol{z}_{\alpha}$ are taken along the TAMD/TAMC sweeping trajectory. The first Gaussian is deposited (centered) at the first point of the trajectory, $\boldsymbol{z}_{1}=\boldsymbol{z}(0)$. The other Gaussians are deposited at the positions $\boldsymbol{z}(t)$ having a distance from the centers of the previously deposited Gaussians, $\left\{\boldsymbol{z}_{\alpha}\right\}_{\alpha=1, L}$, greater than a predetermined value $d$, $\min _{\alpha}\left(\left|\boldsymbol{z}(t)-\boldsymbol{z}_{\alpha}\right|\right) \geqslant d$. $d$ is a parameter controlling the size of the basis set (number of functions in the basis set), and thus the accuracy of the reconstructed Landau free energy.

As mentioned above, we set the values of the expansion coefficients $\boldsymbol{c}$, and $\boldsymbol{\sigma}$, via the least square fitting of the gradient of the Landau free energy estimated by $\mathrm{RMC} / \mathrm{RMD}$. To this end we introduce the objective function:

$$
\begin{aligned}
E(\boldsymbol{c}, \boldsymbol{\sigma}) & =\sum_{k}\left|\nabla_{\boldsymbol{z}} \tilde{F}_{\boldsymbol{\xi}}^{c, \boldsymbol{\sigma}}\left(\boldsymbol{z}_{k}\right)-\tilde{\boldsymbol{f}}\left(\boldsymbol{z}_{k}\right)\right|^{2} \\
& =\sum_{k}\left|\sum_{\alpha}\left\{c_{\alpha} \boldsymbol{\nabla} \chi_{\alpha}\left(\boldsymbol{z}_{k} ; \boldsymbol{\sigma}\right)\right\}-\tilde{\boldsymbol{f}}\left(\boldsymbol{z}_{k}\right)\right|^{2}
\end{aligned}
$$

with $\tilde{\boldsymbol{f}}\left(\boldsymbol{z}_{k}\right)$ an estimate of $f_{i}(\boldsymbol{z})=\frac{\int d \boldsymbol{r} \kappa_{i}\left(\xi_{i}(\boldsymbol{r})-z_{i}\right) \exp \left\{-\beta\left[V(\boldsymbol{r})+\sum_{i=1, M} \frac{\kappa_{i}}{2}\left(\xi_{i}(\boldsymbol{r})-z_{i}\right)^{2}\right]\right\}}{\int d \boldsymbol{r} \exp \left\{-\beta\left[V(\boldsymbol{r})+\sum_{i=1, M} \frac{\kappa_{i}}{2}\left(\xi_{i}(\boldsymbol{r})-z_{i}\right)^{2}\right]\right\}}$ (see Eq. (12)). The optimal values of $\boldsymbol{c}$ and $\boldsymbol{\sigma}$ are:

$$
\left\{\boldsymbol{c}^{*}, \boldsymbol{\sigma}^{*}\right\}=\operatorname{argmin}_{\boldsymbol{c}, \sigma} E(\boldsymbol{c}, \boldsymbol{\sigma}) .
$$

This minimization is, in general, a complex non-linear problem. However, if $\sigma_{\alpha}$ is the same for all the $\chi_{\alpha}(\boldsymbol{z} ; \boldsymbol{\sigma})$ functions (hereafter simply denoted by $\sigma$ ), a restriction implied in the definition of the basis set given above requiring a validation, the problem can be simplified. We can use a two-step procedure. First, for a given value of $\sigma$ we minimize $E(\boldsymbol{c}, \sigma)$ only with respect to $\boldsymbol{c}$, thus obtaining a solution parametrically depending on the value of $\sigma, c^{*}(\sigma) \cdot c^{*}(\sigma)$ (and $E\left(c^{*}(\sigma), \sigma\right)$ ) are computed for increasing values of $\sigma$ starting from $\sigma=d$, the distance between the centers. Then, the optimal value of $\sigma, \sigma^{*}=\operatorname{argmin}_{\sigma} E\left(c^{*}(\sigma), \sigma\right)$, is identified with the value corresponding to the lowest $E\left(c^{*}(\sigma), \sigma\right)$ among those obtained in the previous step.

At fixed $\sigma, c^{*}$ can be obtained according to the following equation:

$$
\left.\nabla_{\boldsymbol{c}} E(\boldsymbol{c}, \sigma)\right|_{\boldsymbol{c}=\boldsymbol{c}^{*}}=\mathcal{A} \boldsymbol{c}^{*}-\boldsymbol{b}=\mathbf{0}
$$

where $\mathcal{A}$ is a matrix of elements $\mathcal{A}_{\alpha, \beta}=\sum_{k} \boldsymbol{\nabla}_{\boldsymbol{z}}^{T} \chi_{\alpha}\left(\boldsymbol{z}_{k}\right) \cdot \boldsymbol{\nabla}_{\boldsymbol{z}} \chi_{\beta}\left(\boldsymbol{z}_{k}\right)$ (the index $T$ denotes the transpose of the vector $\left.\boldsymbol{\nabla}_{\boldsymbol{z}} \chi_{\alpha}\left(\boldsymbol{z}_{k}\right)\right)$ and $b_{\beta}=\sum_{k} \tilde{f}^{T}\left(\boldsymbol{z}_{k}\right) \cdot \boldsymbol{\nabla}_{\boldsymbol{z}} \chi_{\beta}\left(\boldsymbol{z}_{k}\right)$. Eq. (23) can be solved by standard linear algebra methods implemented in widely used libraries of computer routines, available in many programming languages. 
The version of the method explained above assumes that a set of spherical Gaussian functions is adeguate to expand the Landau free energy. However, when the CVs are very different in nature, and/or when the Landau free energy has strong asymmetries, i.e. it is steeper along some direction and shallower along others, the choice of spherical Gaussians is unjustified and non optimal. The standard SSM can be improved, ISSM [32], by using the more general Gaussian basis set: $\chi_{\alpha}(\boldsymbol{r})=\exp \left[-1 / 2\left(\boldsymbol{z}-\boldsymbol{z}_{\alpha}\right)^{T} \Sigma_{\alpha}^{-2}\left(\boldsymbol{z}-\boldsymbol{z}_{\alpha}\right)\right]$, where $\Sigma_{\alpha}$ is the covariance matrix associated to $\chi_{\alpha}(\boldsymbol{r})$ (in the following the symbol $\boldsymbol{\Sigma}$ denotes the array whose elements are $\Sigma_{\alpha}$ matrices).

A second aspect in which ISSM improves over the standard SSM is in the definition of the objective function. In ISSM $E(\boldsymbol{c}, \boldsymbol{\Sigma}) \equiv \sum_{k} w_{k}\left|\tilde{F}_{\boldsymbol{\xi}}^{\boldsymbol{c}, \boldsymbol{\Sigma}}\left(\boldsymbol{z}_{k}\right)-\tilde{\boldsymbol{f}}\left(\boldsymbol{z}_{k}\right)\right|^{2} \cdot w_{k}$ is a weight measuring the relevance of the point $\boldsymbol{z}_{k}$ in the fitting procedure. Thus, for example, setting $w_{k}=1 /\left(\left|\tilde{\boldsymbol{f}}\left(\boldsymbol{z}_{k}\right)\right|^{2}+\delta\right)$, where $\delta$ is a small constant parameter added to avoid singularities, we can increase the relevance of free energy minima (metastabilities) and maxima (transition states) in the least square fitting.

The minimization of the new objective function, $E(\boldsymbol{c}, \boldsymbol{\Sigma})$, cannot be achieved with the simple procedure outlined above for the standard SSM. In this case we use a MC simulated annealing-like procedure [20] in the space of the parameters $c$ and $\boldsymbol{\Sigma}$. This procedure is based on the PDF $p(\boldsymbol{c}, \boldsymbol{\Sigma})=\exp [-\bar{\beta} E(\boldsymbol{c}, \boldsymbol{\Sigma})] / \mathcal{N}$, where $\mathcal{N}$ is a normalization constant and $\bar{\beta}$ is a fictitious inverse temperature. $\bar{\beta}$ is initially set to a relatively low value, and is then increased during the procedure. In principle, when $\bar{\beta}$ is very large the distribution is peaked around the absolute minimum of $E(\boldsymbol{c}, \boldsymbol{\Sigma})$, which then gives the optimal values of $\boldsymbol{c}$ and $\boldsymbol{\Sigma}$. However, depending on the rate of increase of $\bar{\beta}$, the trajectory in the $\boldsymbol{c}-\boldsymbol{\Sigma}$ space can get trapped into a local minimum of $E(\boldsymbol{c}, \boldsymbol{\Sigma})$. Thus, the simulated annealing procedure is repeated several times starting from different initial conditions to confirm that one has found the absolute minimum of the objective function. It is worth remarking that the simulated annealing procedure is computationally cheap with respect to the very expensive calculation of $\tilde{\boldsymbol{f}}\left(\boldsymbol{z}_{k}\right)$. Thus repeating the simulated annealing procedure a few times does not increase significantly the overall computational cost of the simulation.

\section{Applications}

TAMD, TAMC and SSM have been applied by us and several collaborators to various problems including phase transitions [24,39], defects in nanoparticles [14,18]; chemical reactions $[6,31,46]$, biological processes, [26] and the wetting of textured surfaces [8-11]. We have also employed the RMD/RMC method for sampling the conditional PDF of Sect. 2.1.1 as one of the ingredients for our method, the dynamical nonequilibrium MD, for studying non-equilibrium phenomena [5, 37, 38, 44].

In this section we present two applications for elucidating TAMC and SSM. These results have been already reported in previous publications, and are used here only as illustrations of the methods described in the previous section. In Sect. 3.1 we illustrate the use of TAMC to investigate the nucleation of a Lennard-Jones crystal from a moderate supercooled liquid [4]. In Sect. 3.2 we show an application of SSM to reconstruct the free energy landscape of the hydrogen vacancy migration in defected alanates $[33,34]$.

\subsection{Nucleation of a Lennard-Jones crystal from a moderate supercooled liquid}

The formation of a new phase, $B$, into a pre-existing one, $A$, is typically described in terms of an observable measuring the size of the growing nucleus, for example its 
volume, $V_{B}$, or number of particles in the new phase, $N_{B}$. In this model, known as Classical Nucleation Theory (CNT - see Ref. [19] for an exhaustive description of the theory), the free energy of the biphasic system is the sum of two terms: i) a bulk term, $\left(\mu_{B}-\mu_{A}\right) N_{B}$ with $\mu$ chemical potential, and ii) a surface term, $c \gamma_{A, B} N_{B}^{2 / 3}$, with $\gamma_{A, B}$ interface energy between the phases $A$ and $B$. The value of $c$ depends on the shape of the $B$ nucleus; for a spherical nucleus of molecular volume $\bar{v}, c=(36 \pi)^{1 / 3} \bar{v}^{2 / 3}$. Several works have shown that $N_{B}$ is insufficient to describe the nucleation also in the case of a simple, Lennard-Jones (LJ), liquid (see, for example, Ref. [35]). Nevertheless, $N_{B}$ (or equivalent $\mathrm{CVs}$ ) resulted to be a key $\mathrm{CV}$ for describing practically any phase transition.

The definition of the $\mathrm{CV} N_{B}$ is non trivial. First one needs to identify particles in a "state" consistent with the phase $B$ and then find clusters of these particles. In the case of the nucleation of a LJ crystal, we define particles to be crystal-like on the base of their bond orientational order parameter [45]

$$
q_{l m}(i)=\sum_{j=1}^{N_{i}} Y_{l m}\left(\hat{\boldsymbol{r}}_{i j}\right)
$$

where $Y_{l m}\left(\hat{\boldsymbol{r}}_{i j}\right)$ is the spherical harmonics of order $l$ and $m$ computed at the polar and azimuthal angles associated to the vector $\boldsymbol{r}_{i j}$ connecting particles $i$ and $j\left(\hat{\boldsymbol{r}}_{i j}\right.$ is the corresponding unit vector). $N_{i}$ is the number of nearest neighbors of the atom $i$, i.e. the number of particles $j$ satisfying the condition $\left|\boldsymbol{r}_{i j}\right| \leqslant 1.5 \sigma$, with $\sigma$ length parameter of the LJ pair potential.

In crystals the $q_{l m}$ of all atoms is the same because their neighboring atoms have the same relative position $\left(\hat{\boldsymbol{r}}_{i j}=\hat{\boldsymbol{r}}_{i^{\prime} j^{\prime}}\right)$. In liquids, on the contrary, the orientation of $\hat{\boldsymbol{r}}_{i j}$ vectors around each atom is random, and thus $q_{l m}(i)$ of neighboring atoms can differ significantly. On the basis of this empirical observation we distinguish between crystal-like and liquid-like particles. We compute

$$
C_{i j}=\frac{\left|\sum_{m=-l}^{l} q_{l m}(i)^{*} q_{l m}(j)\right|}{\left|q_{l}(i)\right|\left|q_{l}(j)\right|}
$$

with $\left|q_{l}(i)\right|\left(\left|q_{l}(j)\right|\right)$ defined as $\left|q_{l}(i)\right|=\sqrt{\sum_{m=-l}^{l} q_{l m}(i)^{*} q_{l m}(i)} . C_{i j}$ can be interpreted as the normalized dot product between the vectors $q_{l}(i)$ and $q_{l}(j)$, with components $q_{l m}(i)$ and $q_{l m}(j)$. Therefore $C_{i j}$ measures how much the two vectors are parallel. In a Bravais lattice at $0 \mathrm{~K}$ the $q_{l m}(i)$ of all the atoms is the same, and $C_{i j}=1$. At finite temperature this is not true, but it has been empirically observed that $C_{i j} \geqslant 0.5$ [47]. We call particles $i$ and $j$ satisfying this condition "connected". In the following, this term will be used in this strict sense. Particles satisfying this condition are found also in liquids, but the number of connected particles in LJ crystals $(\geqslant 7)$ is higher than in the liquid at the same $T(\leqslant 4-5)$. Thus, we use the number of particles connected to the particle $i, n_{i}$, to assign it to the crystal-like set.

The next step is to identify clusters of crystal-like particles. These are set of connected crystal-like particles. For this we use the Depth-First search method [22]. This method consists in searching the crystal-like particles connected to a root one, selected randomly, and exploring as far as possible along these connections. Once all the particles directly or indirectly connected to the root are identified the search for the members of the present cluster is completed. The search for the particles belonging to another cluster is then started by defining a new root among the crystallike particles not yet assigned to any nucleus. This search is repeated until all the crystal-like particles have been assigned to a cluster. Our CV, $N_{B}(\boldsymbol{r})$, is the size (number of particles) of the biggest of these clusters. 

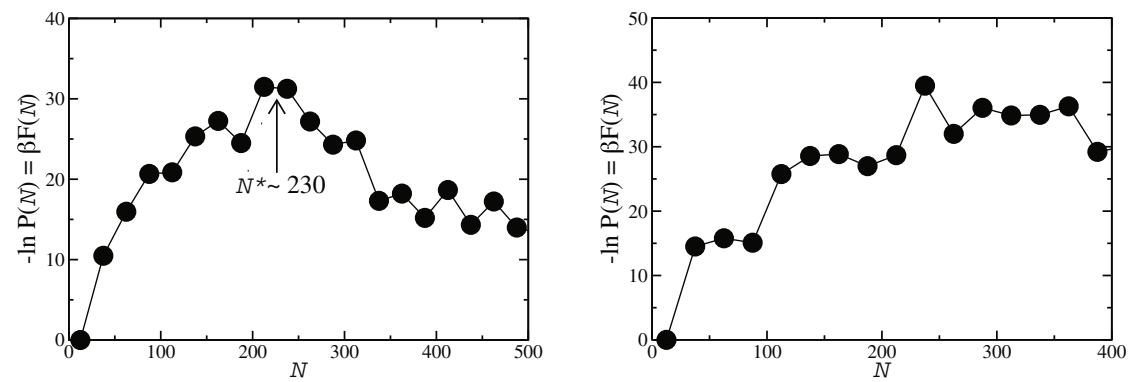

Fig. 2. Left: free energy vs. $N_{A}$ curve at $P=5.6$ and $T=0.92$. The nucleus critical size is also reported. Right: same graph at $T=1.2$. Adapted from Ref. [4].

Being the value of $N_{B}(\boldsymbol{r})$ the result of a search algorithm, this CV is not differentiable and it cannot be used with MD based enhanced sampling methods. TAMC allows to overcome this problem. It is worth remarking that for the reconstruction of the Landau free energy of the single $N_{B}(\boldsymbol{r})$ one could use simpler methods, e.g. umbrella sampling. However, in more realistic cases, when one consider more than one $\mathrm{CV}$, and/or other metastabilities might be present (e.g. various crystal phases), the umbrella sampling is unsuitable, and TAMC becomes a convenient method to explore the CV-space.

We performed unbiased $2 \times 10^{6}$ steps MC of a liquid sample of $3456 \mathrm{LJ}$ particles at pressure and temperature $P=5.6$ and $T=0.92$ (LJ units are used throughout this section), corresponding to a $17 \%$ supercooling (current temperature is $17 \%$ lower than the crystallization temperature). Along this simulation we computed $N_{B}(\boldsymbol{r})$. We observed $N_{B}(\boldsymbol{r})$ fluctuating around 20, which is smaller than the size corresponding to the maximum of the Landau free energy (see below). This result simply confirms that nucleation is a rare event, and that relevant information on this process, such as the Landau free energy barrier and the critical size of the nucleus, cannot be obtained by brute force simulations in the conditions of "moderate" supercooling.

We, then, run a TAMC simulation at $\bar{\beta}=1 /\left(30 k_{B} T\right)$, to be compared with a previous estimate of the Landau free energy barrier at $T=0.83$ of $\Delta W^{\dagger} \sim 25 k_{B} T$ [35]. This TAMC trajectory explores a broad range of values of the CV in the interval $N_{A}(\boldsymbol{r}) \in[0-600]$. To reconstruct the Landau free energy profile and take advantage of the ICHEC Stokes supercomputer, with its large number of cores, we run 32 independent trajectories started from 4 configurations at $N_{B}(\boldsymbol{r})=100,200,300,400$. For each configuration we extracted 8 initial velocities $\dot{z}$ from a Maxwell-Boltzmann distribution at $\bar{T}=30$. In Fig. 2 (left) we report the corresponding free energy profile.

In spite of the difference in the free energy barrier, the critical size as estimated from our TAMC simulation is in good agreement with the one reported by Moroni et al. [35]. The critical size, defined as the $z$ corresponding to the maximum of the free energy, is $\sim 230$ in our simulations while it is 243 in Ref. [35].

We also run simulations at a higher temperature, $T=1.2$ (Fig. 2, right). According to the LJ phase diagram [15], at this temperature the most stable phase is the liquid phase. We therefore expect the Landau free energy curve to increase monotonically with the nucleus size. Our simulations produce a result qualitatively consistent with this trend: the Landau free energy increases with the nucleus size, even though it seems to reach a plateau at large values of $N_{B}$. This is most likely due to the insufficient statistics we get for states of very low probability.

We also analyzed the structure of nuclei formed along the TAMC trajectory. Focusing on the $T=0.92$ case, we found that sub-critical (small) nuclei are, globally, disordered. An example of such a nucleus is shown in Fig. 3A. This nucleus does not 


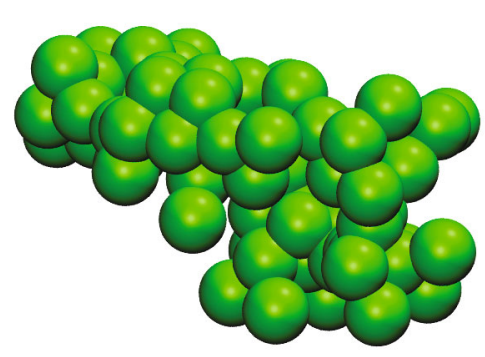

(A)

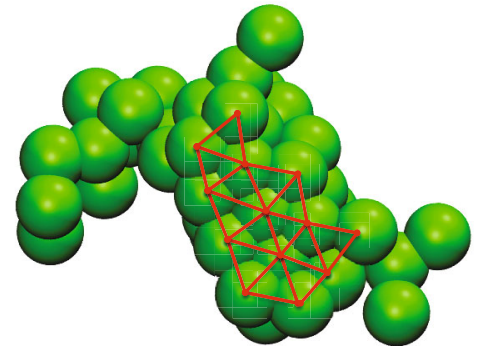

(B)

Fig. 3. Snapshots of an under-critical nucleus (A) and the ordered core of a post-critical nucleus (B). In panel (B) the bonds are superimposed to the atomistic structure to emphasize the ordered shape of the structure. Adapted from Ref. [4]

show any degree of global ordering. Rather, it looks liquid-like. On the contrary, postcritical nuclei show a more ordered structure, with an fcc/hcp-like core (see Fig. 3B).

\subsection{Hydrogen diffusion in a defected sodium alanate crystal}

After discovery that Ti-doped sodium alanates can reversibly uptake/release hydrogen [2] $\mathrm{Na}_{n} \mathrm{AlH}_{m}$ became a prototypical material for hydrogen storage.

In the experimental investigation of the de-hydrogenation process of $\mathrm{NaAlH}_{4}$ a controversy emerged. This concerns the nature of a mobile species present in $\mathrm{Na}_{3} \mathrm{AlH}_{6}$, one of the products of dissociation of $\mathrm{NaAlH}_{4}$

$$
3 \mathrm{NaAlH}_{4} \leftrightharpoons \mathrm{Na}_{3} \mathrm{AlH}_{6}+2 \mathrm{Al}+3 \mathrm{H}_{2} .
$$

Anelastic spectroscopy experiments $[41,42]$ have identified this mobile species to be hydrogen related, and involved in a process characterized by an activation barrier of $\sim 0.12 \mathrm{eV}$. It has been speculated that this mobile species is an hydrogen vacancy diffusing in the forming $\mathrm{Na}_{3} \mathrm{AlH}_{6}$ crystal. Successively, quasi-elastic neutron scattering experiments [53] supported the hypothesis that the mobile species was an Na vacancy. With the aim to clarify this question we reconstructed the free energy of a set of CVs, described below, suitable to describe the H-vacancy migration process.

Vacancy migration is an elusive process, and several CVs have been introduced to describe it $[1,7,12,40]$. Apart for the method introduced in Ref. [7], which has been developed after the investigation we report here, all the other methods assume the main involvement of one single atom in the process. In the present case, we cannot exclude the involvement of more atoms in a cooperative process. Thus, we did not use any of the CVs introduced in previous works. As CVs we use the smoothed coordination number of the $\mathrm{Al}$ atoms of the sample with respect to hydrogen. Given the configuration, $\boldsymbol{r}$, the coordination number of the $\mathrm{Al}$ atom $i, C_{A l_{i}}$, is defined as the number of hydrogen atoms at a distance from this aluminum atom lower than a prescribed value, $R$ :

$$
C_{A l_{i}}=\sum_{j=1}^{N_{H}} \Theta_{R}\left(r_{i j}\right)
$$

where the sum runs over the hydrogen atoms in the sample, $r_{i j}$ is the distance between the $i$-th $\mathrm{Al}$ the $j$-th $\mathrm{H}$ atoms, and $\Theta_{R}\left(r_{i j}\right)$ is the Heaviside step function, taking value 


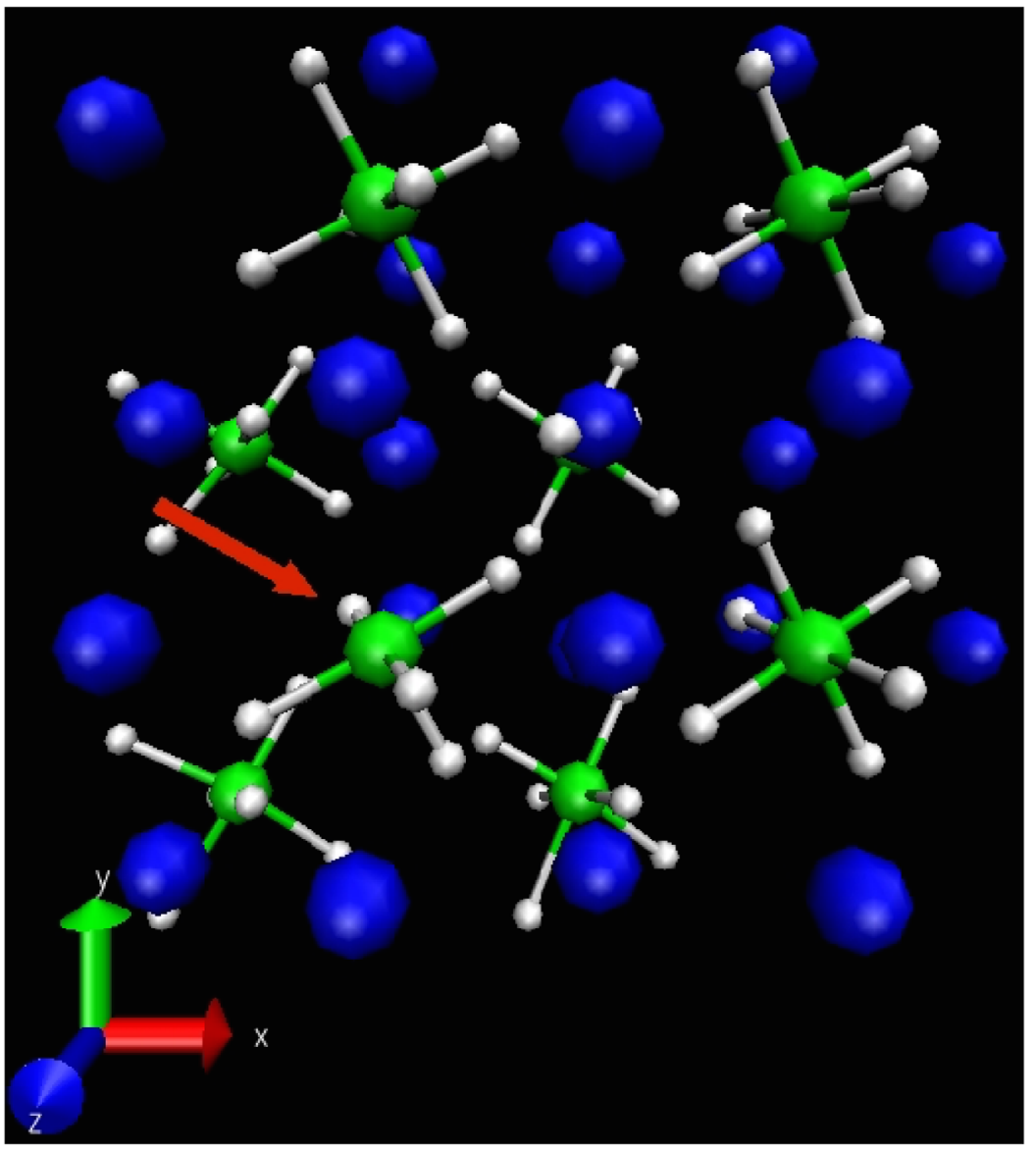

Fig. 4. Computational sample used for the investigation of the H-vacancy migration in defective $\mathrm{NaAlH}_{6}$. Green spheres represent $\mathrm{Al}$ atoms, small white sphere $\mathrm{H}$ atoms, and big blue spheres $\mathrm{Na}$ atoms. The red arrow points the defective $\mathrm{AlH}_{5}$ unit. Adapted from Refs. [33] and [34].

1 if $r_{i j} \leqslant R$ and 0 otherwise. The smoothed version of the coordination number defined in Eq. (27) is obtained by replacing $\Theta_{R}\left(r_{i j}\right)$ with the Fermi function $f\left(r_{i j}\right)=$ $1 /\left(1+\exp \left[\lambda\left(r_{i j}-R\right)\right]\right)$, where $\lambda$ is a parameter controlling the smoothness of $C_{A l_{i}}$. At variance with the standard coordination number of Eq. (27), the smoothed version can take non-integer values.

For a suitable choice of $R$ and $\lambda\left(R=2 \AA\right.$ and $\lambda=10 \AA^{-1}$ in the present case), in a perfect $\mathrm{Na}_{3} \mathrm{AlH}_{6}$ crystal the coordination number of all the $\mathrm{Al}$ atoms is 6 . If the crystal contains $\mathrm{H}$-vacancies, some of the $\mathrm{Al}$ atoms have a lower coordination number, typically 5 . Thus, when an $\mathrm{H}$-vacancy migrates we must observe the corresponding $C_{A l_{i}}$ changing from 6 to 5 , or vice versa, depending on whether the $i$-th $\mathrm{Al}$ atom is an acceptor or donor of the vacancy. The H-vacancy migration process could be a cooperative (collective) phenomenon, with more than two $\mathrm{Al}$ atoms involved in the process. In this case, we should see the coordination number of more than two $\mathrm{Al}$ atoms to change at the same time.

We investigated the $\mathrm{H}$-vacancy migration process in a sample consisting of $7 \mathrm{AlH}_{6}$ and $1 \mathrm{AlH}_{5}$ units (see Fig. 4). Apart for the biasing term, the forces acting on the atoms are obtained from the density functional theory (DFT). We refer the reader 


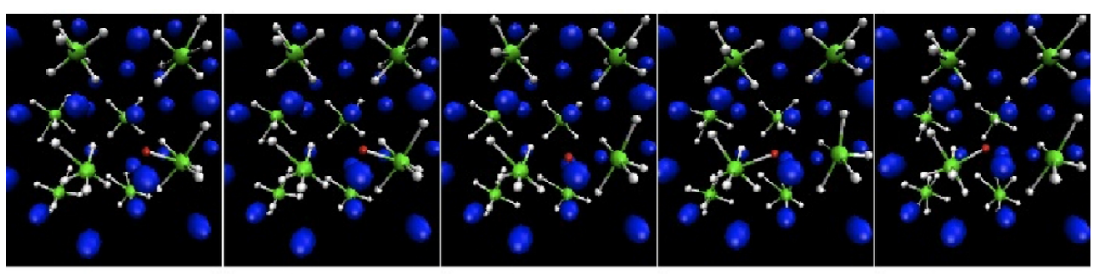

Fig. 5. Series of snapshots along an H-vacancy migration event. In red is highlighted the migrating hydrogen atom. Adapted from Refs. [33] and [34].

interested in the details of our simulations to the original articles [33,34]. We first performed a sweeping TAMD simulation, with the temperature of the particles and CVs set to $T=380 \mathrm{~K}$ and $T=6500 \mathrm{~K}$, respectively. In a 100 ps TAMD simulation we observed 44 vacancy migration events. All these events involve only two $\mathrm{Al}$ atoms at a time, with the donation of one of the $\mathrm{H}$ atoms coordinating an aluminum to the other involved in the event. A series of snapshots taken along one of these events is shown in Fig. 5, illustrating the non-cooperative nature of the process.

The fact that the migration involves only two $\mathrm{Al}$ atoms at a time suggests that the migration process can be described in terms of only two coordination numbers, denoted $C_{1}$ and $C_{2}$ in the following, and the corresponding free energy.

We computed the free energy, $W_{C_{1}, C_{2}}\left(z_{1}, z_{2}\right)$, using different versions of the SSM: i) the normal version, ii) the version with the normal objective function but with a different scalar value of $\sigma$ per Gaussian function in the basis set, and iii) the version with the weighted objective function and a different scalar value of $\sigma$ per Gaussian function in the basis set. In all the cases we considered two basis sets consisting of 37 and 55 Gaussian functions centered at points taken along the TAMD simulation, as explained in Sect. 2.2. In the case iii) the contribution of each term to the objective function is weighted by $w_{k}=1 /\left(\left|\tilde{\boldsymbol{f}}\left(\boldsymbol{z}_{k}\right)\right|^{2}+\delta\right)$, where $\tilde{\boldsymbol{f}}\left(\boldsymbol{z}_{k}\right)$ is an estimation of the gradient of the Landau free energy at the point $\boldsymbol{z}_{k}$ obtained by RMD, and $\delta$ is a small positive number introduced to avoid singularities.

In Fig. 6 we report the isocontour plots of the two-dimensional free energy function reconstructed with the three approaches. In each panel we report the plots obtained with the two basis sets, in black those corresponding to the 37-elements basis set and in red those corresponding to the 55-elements one. The general features of the free energy reconstructed with the various versions of SSM and with all the basis sets are consistent: two minima at $(\sim 5.7, \sim 4.8)$ and $(\sim 4.8, \sim 5.7)$, and a first order saddle point at $(\sim 5.1, \sim 4.9)$. We notice that in passing from version i) to ii) to iii) there is a systematic improvement of the consistency of the results between the smaller and larger basis set. In particular, we notice a remarkably good agreement between the results obtained with the two basis sets for the version iii) in the region of relatively low free energy, namely in the regions around the two minima and the saddle point. This is the most important region of the CV-space because is the region where most of the reactive trajectories pass through (see Ref. [27], and our review on rare event methods Ref. [3]). The improved convergence of the free energy reconstruction for smaller basis sets for the versions ii) and iii) of SSM is due to the fact that we can find optimal values of $\sigma$ for both the shallower regions of the free energy in correspondence of the minima and saddle point, and the steeper zones in the high energy domain of the free energy. The further improvement in the reconstruction of the Landau free energy obtained with the version iii) in the region of minima and saddle point is due to the higher contribution of the terms associated with these domains to the objective function. 

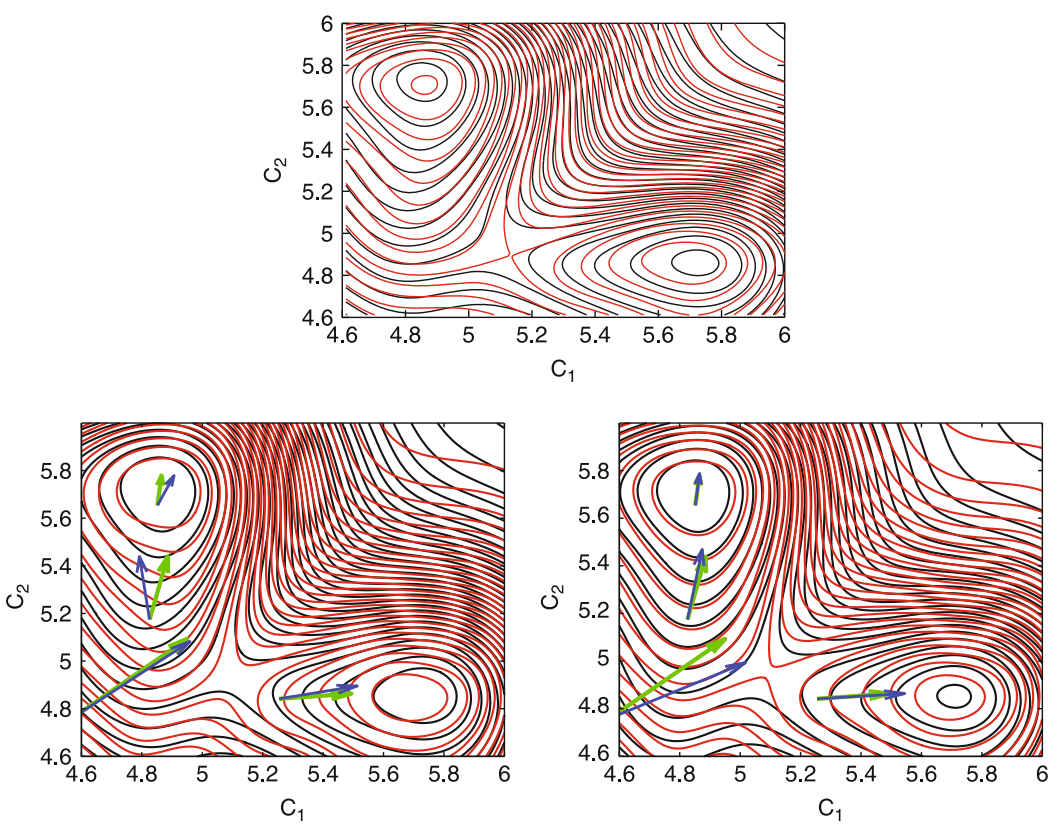

Fig. 6. Comparison of the contour lines of the free energy reconstructed with 37- (black) and 55-elements (red) basis sets. The upper panel shows the profile reconstructed with the standard SSM. The runs in the lower panels were performed using the version with one value of $\sigma$ per Gaussian function and the standard objective function (left) and the weighted objective function (right), respectively. The green arrows in the bottom panels represent the "exact" gradient of the free energy at selected centers. The blue arrows are the gradients computed at the same centers from the reconstruction of the free energy obtained by the various SSM versions. Adapted from Ref. [32].

This latter remark is made more evident by the comparison between the exact gradient of the free energy, obtained by RMD (Sect. 2.1.1), and the gradient of the reconstructed free energy. We notice that in the case of the standard objective function the agreement between the exact and reconstructed gradient of the free energy is very good in the high free energy domain, where its gradient is large (long arrows at the left bottom of the graphs). On the contrary, the agreement is worse in the relative low free energy region. This is because the points where the gradient is large give a larger contribution to the objective function. This problem is solved with the weighted objective function, and we notice that in this case the agreement is improved in the relevant low free energy domain.

\section{Conclusions}

In this article we discussed a set of methods for exploring the collective variable space and reconstruct the Landau free energy in presence of metastabilities. In principle, TAMD and TAMC can be used for both exploring the CV space and, via binning-andhistogramming, compute the Landau free energy in this space. However, binning-andhistogramming is not an efficient approach, and SSM is better suited at reconstructing the free energy in the "reactive region".

It is worth remarking that the methods discussed in this article cannot be used as black boxes for several reasons. First of all, these methods require the selection of a 
suitable set of CVs. This problem, which is not specific of the methods discussed here but is common to all the collective variable methods, is very difficult to tackle as, at the moment, there are not simple procedures to identify the relevant CVs a priori. There are attempts to address this issue (see, for example, Refs. [16, 25,43,49]), but much remains to be done in this direction. Second, TAMD/TAMC and SSM require the tuning of a set of parameters, such as the masses and value of the frictions in TAMD (or characteristic times of the Nosé-Hoover thermostat, if these EoMs are used in place of the overdamped dynamics), the coupling constants $\boldsymbol{\kappa}$ of the restraining potential for the calculation of the mean force, etc. Thus, some experience and extensive testing is necessary to obtain a reliable description of the reactive process and a correct estimate of the free energy landscape.

SM and GC thank Science Foundation Ireland (SFI) for support via the grant SFI 08-IN.1I1869. S.M. acknowledges financial support from the MIUR-FIRB Grant No. RBFR10ZUUK.

\section{A Potential of mean force and the Landau free energy}

In this appendix we show that $-\nabla_{\boldsymbol{\xi}} W_{\boldsymbol{\xi}}(z)$ is the expectation value of a generalized mean force computed over a conditional "equilibrium" distribution. Thus, the integral of $-\nabla_{\boldsymbol{\xi}} W_{\boldsymbol{\xi}}(z)$ along a path in the $\boldsymbol{\xi}$-space is the work made by the generalized forces during a reversible process bringing the system from the initial to the final point, i.e. a reversible work, thus a free energy.

To prove that $-\nabla_{\boldsymbol{\xi}} W_{\boldsymbol{\xi}}(z)$ is a generalized mean force we use Eqs. (8) and the definition of the reduced PDF

$$
p_{\boldsymbol{\xi}}(\boldsymbol{z})=\int d \boldsymbol{\Gamma} m(\boldsymbol{\Gamma}) \boldsymbol{\delta}(\boldsymbol{\xi}(\boldsymbol{\Gamma})-\boldsymbol{z})
$$

with $\boldsymbol{\delta}(\boldsymbol{\xi}(\boldsymbol{\Gamma})-\boldsymbol{z})=\prod_{i=1, M} \delta\left(\xi_{i}(\boldsymbol{\Gamma})-z_{i}\right)$. First, we introduce a set of generalized coordinates and associated momenta, $\left(\boldsymbol{\xi}, \boldsymbol{q}, \boldsymbol{p}_{\boldsymbol{\xi}}, \boldsymbol{p}_{\boldsymbol{q}}\right)$, where $\boldsymbol{\xi}$ are the CVs considered in the main text of the article, and $\boldsymbol{q}, \boldsymbol{p}_{\boldsymbol{\xi}}$ and $\boldsymbol{p}_{\boldsymbol{q}}$ are the variables and associated momenta necessary to complete the canonical transformation from the original set of variables $(\boldsymbol{r}, \boldsymbol{p})$. For a canonical transformation holds the relation $d \boldsymbol{\Gamma} m(\boldsymbol{\Gamma})=d \boldsymbol{\xi} d \boldsymbol{q} d p_{\boldsymbol{\xi}} d p_{\boldsymbol{q}} p\left(\boldsymbol{\xi}, \boldsymbol{q}, \boldsymbol{p}_{\boldsymbol{\xi}}, \boldsymbol{p}_{\boldsymbol{q}}\right)$, where $p\left(\boldsymbol{\xi}, \boldsymbol{q}, \boldsymbol{p}_{\boldsymbol{\xi}}, \boldsymbol{p}_{\boldsymbol{q}}\right)$ is the PDF at a point in the $\left(\boldsymbol{\xi}, \boldsymbol{q}, \boldsymbol{p}_{\boldsymbol{\xi}}, \boldsymbol{p}_{\boldsymbol{q}}\right)$ space. In the following we assume to work in the canonical ensemble. In this case

$$
\frac{d \boldsymbol{\Gamma} \exp [-\beta \mathcal{H}(\boldsymbol{\Gamma})]}{\int d \boldsymbol{\Gamma} \exp [-\beta \mathcal{H}(\boldsymbol{\Gamma})]}=\frac{d \boldsymbol{\xi} d \boldsymbol{q} d p_{\boldsymbol{\xi}} d p_{\boldsymbol{q}} \exp \left[-\beta \overline{\mathcal{H}}\left(\boldsymbol{\xi}, \boldsymbol{q}, \boldsymbol{p}_{\boldsymbol{\xi}}, \boldsymbol{p}_{\boldsymbol{q}}\right)\right]}{\int d \boldsymbol{\xi} d \boldsymbol{q} d p_{\boldsymbol{\xi}} d p_{\boldsymbol{q}} \exp \left[-\beta \overline{\mathcal{H}}\left(\boldsymbol{\xi}, \boldsymbol{q}, \boldsymbol{p}_{\boldsymbol{\xi}}, \boldsymbol{p}_{\boldsymbol{q}}\right)\right]}
$$

with $\left.\left.\overline{\mathcal{H}}\left(\boldsymbol{\xi}, \boldsymbol{q}, \boldsymbol{p}_{\boldsymbol{\xi}}, \boldsymbol{p}_{\boldsymbol{q}}\right)\right]=\mathcal{H}\left(\boldsymbol{\Gamma}\left(\boldsymbol{\xi}, \boldsymbol{q}, \boldsymbol{p}_{\boldsymbol{\xi}}, \boldsymbol{p}_{\boldsymbol{q}}\right)\right)\right]$ the system Hamiltonian in the coordinates $\left(\boldsymbol{\xi}, \boldsymbol{q}, \boldsymbol{p}_{\boldsymbol{\xi}}, \boldsymbol{p}_{\boldsymbol{q}}\right)$. Further, we make use of the property of the Dirac- $\delta$ function that $d \delta(x-y) / d x=-d \delta(x-y) / d y$. Plugging all that together we get:

$$
\begin{aligned}
\frac{\partial p_{\boldsymbol{\xi}}(\boldsymbol{z})}{\partial z_{i}} & =\frac{\partial}{\partial z_{i}} \int d \boldsymbol{r} d \boldsymbol{p} m(\boldsymbol{r}, \boldsymbol{p}) \boldsymbol{\delta}(\boldsymbol{\xi}(\boldsymbol{r}, \boldsymbol{p})-\boldsymbol{z}) \\
& =\int d \boldsymbol{\xi} d \boldsymbol{q} d p_{\boldsymbol{\xi}} d p_{\boldsymbol{q}} p\left(\boldsymbol{\xi}, \boldsymbol{q}, \boldsymbol{p}_{\boldsymbol{\xi}}, \boldsymbol{p}_{\boldsymbol{q}}\right) \frac{\partial \boldsymbol{\delta}(\boldsymbol{\xi}-\boldsymbol{z})}{\partial z_{i}} \\
& =-\int d \boldsymbol{\xi} d \boldsymbol{q} d p_{\boldsymbol{\xi}} d p_{\boldsymbol{q}} p\left(\boldsymbol{\xi}, \boldsymbol{q}, \boldsymbol{p}_{\boldsymbol{\xi}}, \boldsymbol{p}_{\boldsymbol{q}}\right) \frac{\partial \boldsymbol{\delta}(\boldsymbol{\xi}-\boldsymbol{z})}{\partial \xi_{i}} .
\end{aligned}
$$


Integrating by parts the r.h.s. of the last equality, one gets

$$
\begin{aligned}
\frac{\partial p_{\boldsymbol{\xi}}(\boldsymbol{z})}{\partial z_{i}} & =\int d \boldsymbol{\xi} d \boldsymbol{q} d p_{\boldsymbol{\xi}} d p_{\boldsymbol{q}} \frac{\partial p\left(\boldsymbol{\xi}, \boldsymbol{q}, \boldsymbol{p}_{\boldsymbol{\xi}}, \boldsymbol{p}_{\boldsymbol{q}}\right)}{\partial \xi_{i}} \boldsymbol{\delta}(\boldsymbol{\xi}-\boldsymbol{z}) \\
& =-\beta \int d \boldsymbol{r} d \boldsymbol{p} \frac{\partial \overline{\mathcal{H}}\left(\boldsymbol{\xi}, \boldsymbol{q}, \boldsymbol{p}_{\boldsymbol{\xi}}, \boldsymbol{p}_{\boldsymbol{q}}\right)}{\partial \xi_{i}} m(\boldsymbol{r}, \boldsymbol{p}) \delta(\boldsymbol{\xi}(\boldsymbol{r}, \boldsymbol{p})-\boldsymbol{z})
\end{aligned}
$$

Plugging Eq. (31) into Eq. (8) we get:

$$
-\frac{\partial W_{\boldsymbol{\xi}}(\boldsymbol{z})}{\partial z_{i}}=-\frac{\int d \boldsymbol{r} d \boldsymbol{p} \frac{\partial \mathcal{H}(\boldsymbol{\Gamma})}{\partial \xi_{i}} m(\boldsymbol{r}, \boldsymbol{p}) \delta(\boldsymbol{\xi}(\boldsymbol{r}, \boldsymbol{p})-\boldsymbol{z})}{p_{\boldsymbol{\xi}}(\boldsymbol{z})} .
$$

Therefore, $-\boldsymbol{\nabla}_{z} W_{\boldsymbol{\xi}}(\boldsymbol{z})$ is the average value of the generalized force $-\nabla_{\boldsymbol{\xi}} \mathcal{H}\left(\boldsymbol{r}(\boldsymbol{\xi}, \boldsymbol{q}), \boldsymbol{p}\left(\boldsymbol{\xi}, \boldsymbol{q}, \boldsymbol{p}_{\boldsymbol{\xi}}, \boldsymbol{p}_{\boldsymbol{q}}\right)\right)$ over the conditional PDF $m(\boldsymbol{r}, \boldsymbol{p} \mid \boldsymbol{z})$ of Eq. (6).

\section{References}

1. C. Bennet, Exact Defect Calculations in Model Substances (Academic Press, 1975), p. 73

2. B. Bogdanovi, S.M., J. Alloys Compounds 1, 253 (1997)

3. S. Bonella, S. Meloni, G. Ciccotti, EPJ B, 85, 97 (2012)

4. G. Ciccotti, S. Meloni, Phys. Chem. Chem. Phys. 13, 5952 (2011)

5. G. Cottone, G. Lattanzi, G. Ciccotti, R. Elber, J. Phys. Chem. B 116, 3397 (2012)

6. A.M. Elena, S. Meloni, G. Ciccotti, J. Phys. Chem. A 117, 13039 (2013)

7. P.-A. Geslin, S. Meloni, G. Ciccotti, J. Chem. Phys. 138, 144103 (2013)

8. A. Giacomello, M. Chinappi, S. Meloni, C.M. Casciola, Phys. Rev. Lett. 109, 226102 (2012)

9. A. Giacomello, M. Chinappi, S. Meloni, C.M. Casciola, Langmuir 29, 14873 (2013)

10. A. Giacomello, S. Meloni, M. Chinappi, C.M. Casciola, Langmuir 28, 10764 (2012)

11. A. Giacomello, S. Meloni, M. M̈̈uller, C. Casciola, J. Chem. Phys. 142, 104701 (2015)

12. J. Gillan, J.H. Harding, R.J. Tarento, J. Phys. C 20, 2331 (1987)

13. H. Grubmüller, Phys. Rev. E 52, 2893 (1995)

14. R. Guerra, M. Ippolito, S. Meloni, S. Ossicini, Appl. Phys. Lett. 100, 181905 (2012)

15. J.-P. Hansen, L. Verlet, Phys. Rev. 184, 151 (1969)

16. B. Hashemian, D. Millán, M. Arroyo, J. Chem. Phys. 139, 214101 (2013)

17. T. Huber, A.E. Torda, W.F. van Gunsteren, J. Comput. Molec. Des. 8, 695 (1994)

18. M. Ippolito, S. Meloni, Phys. Rev. B 83, 165209 (2011)

19. K. Kelton, A.L. Greer, Nucleation in condensed matter: applications in materials and biology, Vol. 15 (Pergamon, 2010)

20. S. Kirkpatrick, C.D. Gelatt, M.P. Vecchi, Science 220, 671 (1983)

21. J.G. Kirkwood, J. Chem. Phys. 3, 300 (1935)

22. D. Knuth, The Art of Computer Programming, Vol. 1, 3rd edition (Addison-Wesley, Boston, 1997)

23. A. Laio, M. Parrinello, Proc. Natl. Acad. Sci. 99, 12562 (2002)

24. M. Lauricella, S. Meloni, N.J. English, B. Peters, G. Ciccotti, J. Phys. Chem. C 118, 22847 (2014)

25. P. Maragakis, A. van der Vaart, M. Karplus, J. Phys. Chem. B 113, 4664 (2009)

26. L. Maragliano, G. Cottone, G. Ciccotti, E. Vanden-Eijnden, J. Amer. Chem. Soc. 132, $1010(2010)$

27. L. Maragliano, A. Fischer, E. Vanden-Eijnden, G. Ciccotti, J. Chem. Phys. 125, 024106 (2006)

28. L. Maragliano, E. Vanden-Eijnden, Chem. Phys. Lett. 426, 168 (2006)

29. L. Maragliano, E. Vanden-Eijnden, J. Chem. Phys. 128, 184110 (2008) 
30. G.J. Martyna, M.L. Klein, M. Tuckerman, J. Chem. Phys. 97, 2635 (1992)

31. M. Monteferrante, S. Bonella, G. Ciccotti, Phys. Chem. Chem. Phys. 13, 10546 (2011)

32. M. Monteferrante, S. Bonella, S. Meloni, G. Ciccotti, Molec. Simul. 35, 1116 (2009)

33. M. Monteferrante, S. Bonella, S. Meloni, E. Vanden-Eijnden, G. Ciccotti, Scientific Model. Simul. SMNS 15, 187 (2008)

34. M. Monteferrante, S. Bonella, S. Meloni, E. Vanden-Eijnden, G. Ciccotti, Calculations of free energy barriers for local mechanisms of hydrogen diffusion in alanates, Vol. 68 (Springer, Berlin, 2009), p. 187

35. D. Moroni, P.R. ten Wolde, P.G. Bolhuis, Phys. Rev. Lett. 94, 235703 (2005)

36. S. Orlandini, S. Meloni, G. Ciccotti, J. Stat. Phys. 145, 812 (2011)

37. S. Orlandini, S. Meloni, G. Ciccotti, Hydrodynamics from dynamical non-equilibrium MD, AIP Conference Proceedings 1332, 77 (2011)

38. S. Orlandini, S. Meloni, G. Ciccotti, Phys. Chem. Chem. Phys. 13, 13177 (2011)

39. S. Orlandini, S. Meloni, L. Colombo, Phys. Rev. B 83, 235303 (2011)

40. E. Paci, G. Ciccotti, J. Phys.: Condens. Mat. 4, 2173 (1992)

41. O. Palumbo, R. Cantelli, A. Paolone, C. Jensen, S. Srinivasan, J. Phys. Chem. B 109, $1168(2005)$

42. O. Palumbo, A. Pauline, R. Cantelli, C. Jensen, M. Sulic, J. Phys. Chem. B 110, 9105 (2006)

43. B. Peters, B.L. Trout, J. Chem. Phys. 125, 054108 (2006)

44. M. Pourali, S. Meloni, F. Magaletti, A. Maghari, C.M. Casciola, G. Ciccotti, J. Chem. Phys. 141, 154107 (2014)

45. P.J. Steinhardt, D.R. Nelson, M. Ronchetti, Phys. Rev. B 28, 784 (1983)

46. F. Sterpone, S. Bonella, S. Meloni, J. Phys. Chem. C 116, 19636 (2012)

47. P. ten Wolde, M. Ruiz-Montero, D. Frenkel, J. Chem. Phys. 104, 9932 (1996)

48. G.M. Torrie, J.P. Valleau, J. Comput. Phys. 23, 187 (1977)

49. G.A. Tribello, M. Ceriotti, M. Parrinello, Proc. Natl. Acad. Sci. 107, 17509 (2010)

50. E. Vanden-Eijnden, Commun. Math. Sci. 1, 385 (2003)

51. J. VandeVondele, U. Rothlisberger, J. Phys. Chem. B 106, 203 (2002)

52. M. Venturoli, E. Vanden-Eijnden, G. Ciccotti, J. Math. Chem. 45, 188 (2009)

53. J. Voss, Q. Shi, H. Jacobsen, M. Zamponi, K. Lefmann, T. Vegge, J. Phys. Chem. B 111, 3886 (2007) 